\title{
AGENTES PEDAGÓGICOS COM DIMENSÕES AFETIVAS: uma tecnologia no apoio ao ensino e a aprendizagem
}

\author{
Cícero C. Quarto ${ }^{1}$, Antônio N. Oliveira ${ }^{2}$, Magda Bercht ${ }^{3}$ \\ ${ }^{1}$ Centro de Ciências Tecnológicas - Universidade Estadual do Maranhão (UEMA) \\ Caixa Postal 09 - São Luís - MA - Brazil \\ ${ }^{2}$ Departamento de Matemática - Universidade Federal do Maranhão (UFMA) \\ Imperatriz, MA \\ ${ }^{3}$ Instituto de Informática - Universidade Federal do Rio Grande do Sul (UFRGS) \\ Caixa Postal 15064 - 91501-970 - Porto Alegre - RS - Brazil \\ cicero@engcomp.uema.br, aneresoliveira@gmailcom, bercht@inf.ufrgs.br
}

\begin{abstract}
This article shows a view of the state of the art of Affective Pedagogical Agents (APA), in aid to teaching and learning supported by technology and virtual environments. The study presents the main features, applications and perspectives for APA, in order to contribute to greater visibility towards directions future in the development and applications of this technology.
\end{abstract}

Resumo. Este artigo mostra uma visão do estado da arte de Agentes Pedagógicos Afetivos(APA) no auxilio ao ensino e a aprendizagem apoiados por tecnologias e ambientes virtuais. $O$ estudo apresenta as principais características, aplicações e perspectivas para APA, de forma a contribuir para uma maior visibilidade rumo a direções futuras no desenvolvimento e aplicações desta tecnologia.

\section{Introdução}

Para Chatzara et al. (2012), comunicação afetiva é relevante na aprendizagem uma vez que pode apoiar e oferecer um sentido de suporte quando ocorrerem afetos negativos. Silva; Figueiredo (2012) consideram que no ensino presencial o professor consegue "sentir" a turma, tanto na dimensão de entendimento dos conteúdos como dos estados afetivos que estão se apresentando, e assim, pode alterar a sua didática. Porém, verificar a dimensão afetiva dos alunos em e-Learning, exige muito mais do professor visto a falta do presencial, sendo toda a informação transcrita em grande volume de dados oriundas de interações produzidas nestes ambientes. Aplicações $e$-Learning referem-se a todos os artefatos que apoiam o ensino e a aprendizagem, como Objetos de Aprendizagem (OA), Sistemas Tutores Inteligentes (STI), Ambientes Virtuais de Aprendizagem (AVA). Muitos destes artefatos se apoiam em tecnologia de agentes que são usados para nomear desde processos simples até grupos ou conjuntos de hardware e/ou software complexos, mas todos denotando uma entidade para executar uma tarefa ou um conjunto destas, autônoma ou semi-autonomamente ( Bercht, 2001). Este trabalho faz uma revisão de APA visando auxiliar no desenvolvimento e aplicação desta 
tecnologia computacional para fins educacionais. $\mathrm{O}$ artigo está estruturado nas seguintes seções: A Seção 2 descreve sobre Dimensão afetiva em APA. A Metodologia de pesquisa é descrita na Seção 3. Análises e discussões são descritas na Seção 4. Já na Seção 5 são feitas as Conclusões e direções futuras, seguidas das Referências.

\section{Dimensão afetiva em agentes pedagógicos}

A Ciência da Computação tem feito estudos incorporando a dimensão afetiva em computadores, determinando-se, assim, a área de Computação Afetiva (CA) (Picard, 1997; Bercht 2001). A CA estuda a inferência da afetividade do humano em interação com sistemas computacionais como se fossem observadores em terceira pessoa, bem como a expressão afetiva de modo melhorar a comunicação entre outras capacitações. Desse modo, se pode entender as formas de manifestação da afetividade (expressões corporal, verbal, escrita, etc) e quando necessário, sintetizá-las. Neste sentido, Jaques (2004), desenvolveu um personagem virtual, chamado PAT (do inglês Pedagogical Agent Tutor) como um agente pedagógico animado. PAT se utiliza de expressões, gestos, e se comunica com alunos ao detectar estados afetivos negativos durante a interação no processo educacional.

\section{Metodologia de pesquisa}

Com base em Flick (2013), foi adotada revisão da literatura e análise qualitativa, com o intuito de conhecer as contribuições em APA, tais como focos, dimensões afetivas atendidas pelos agentes, técnicas utilizadas, visando com isso extrair, analisar e sintetizar dados de estudos no Brasil e no Mundo (ver Figura 1). Por convenção, a coleta de dados orientou-se em descobrir estudos e apresentar seus principais resultados a partir de duas linhas do tempo. Uma antes de 2010 e outra a partir de 2010 a 2014. No Brasil, foi feito um levantamento nos Anais do CSBC, SBIE e WIE e nos Periódicos RENOTE e RBIE. Já no mundo, foram feitas consultas nas bases do IEEE, ACM e MIT para um tamanho $n$ de amostras de artigos consultados de $\boldsymbol{n}_{\text {IEEE }}=86, \boldsymbol{n}_{\text {ACM }}=200 \mathrm{e}$ $\boldsymbol{n}_{\text {MIT }}=300$. Os resultados são mostrados, em forma de resumo para a primeira linha do tempo, e em forma de Gráficos, Tabela e Figura, para a segunda linha do tempo, conforme ilustram os Gráficos 1, 2 e 3, bem como a Tabela 1.

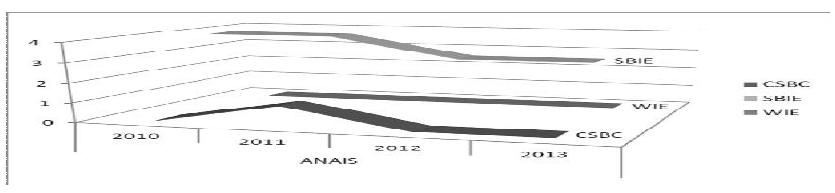

Gráfico 1. № de artigos sobre APA nos Anais CSBC, SBIE e WIE.

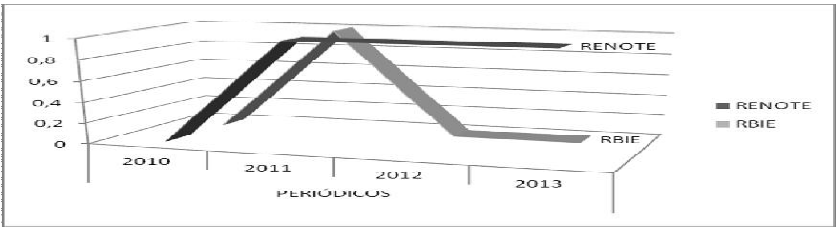

Gráfico 2. № de artigos sobre APA nos Periódicos RBIE e RENOTE. 


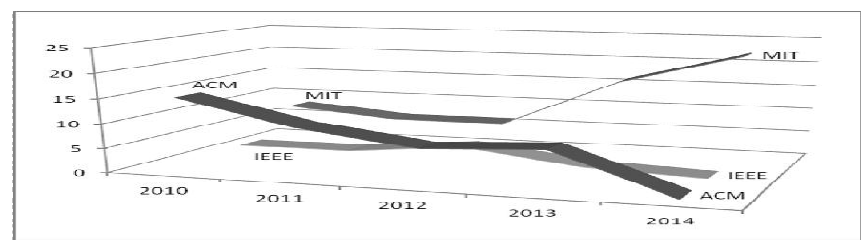

Gráfico 3. № de artigos sobre APA no MIT, IEEE e ACM.

\subsection{Primeira linha do tempo - antes de 2010}

Foi detectado que desde a década de 1997 já existiam pesquisas em APA. As principais são: Johnson; Rickel (1997); Johnson et al. (2000); Santos et al. (2001), Johnson et al. (2003), Jaques (2004); Deters et al. (2006); Strauss; Kipp (2008); Or (2008) e Mello et al. (2009). As linhas de pesquisas foram: (i) Reconhecimento e/ou inferências de emoções do aluno a partir de suas ações. (ii) Acompanhamento da interação do aluno com o STI. (iii) Tutoria de alunos de diversas áreas, tais como medicina, biologia, entretenimento, matemática, física, literatura e química. Houve pesquisas na área de robótica educacional, especificamente de robôs afetivos como o Kismet, desenvolvido no MIT, o Furby, desenvolvido pela Tiger Eletronics dos EUA, o Cãozito Aibo, desenvolvido pela Sony e o robô WBD-1, o qual tem a capacidade de expressar emoções, através de expressões faciais, voz e linguagem natural. Dimensões afetivas desta linha: calma, alegria, tristeza, surpresa, interesse, aborrecimento, zanga, sono, medo, confiança, desgosto, felicidade, alívio, perseverança, satisfação, frustração, gratidão, vergonha, admiração, simpatia, companheirismo, cooperação e ânimo.

\subsection{Segunda linha do tempo - 2010 a 2014}

A partir dos Gráficos 1, 2 e 3, descreve-se, na Tabela 1, os APA, seus aspectos pedagógicos e suas dimensões afetivas. Já na Figura 1, são descritos os descobertos sobre APA nas bases da ACM, IEEE e MIT.

Tabela 1: APA, aspectos pedagógicos e dimensões afetivas.

\begin{tabular}{|c|c|}
\hline Agentes & Aspectos Funcionais e Dimensões Afetivas \\
\hline $\begin{array}{l}\text { PAT2Math (Seffrin et al. } \\
\text { 2010) }\end{array}$ & $\begin{array}{l}\text { Auxilia o aluno nas suas dificuldades de aprendizagem; considera suas emoções, de forma a motivá-lo e engajá-lo em } \\
\text { sua interação com o sistema. }\end{array}$ \\
\hline $\begin{array}{l}\text { Dr. Pierre (De Oliveira et } \\
\text { al. 2010) }\end{array}$ & $\begin{array}{l}\text { É um chatterbot com interação e personalidade para apoiar o ensino e aprendizagem de Psiquiatria e Psicologia, } \\
\text { provendo conhecimento por meio de diálogos mais próximos de interlocutores humanos. }\end{array}$ \\
\hline $\begin{array}{l}\text { Persona-Algo (Lapsen et } \\
\text { al. 2010) }\end{array}$ & $\begin{array}{l}\text { Auxilia o professor e o aluno na disciplina de algoritmos, levando em consideração o nível de conhecimento do aluno, } \\
\text { assim como seus estados afetivos. }\end{array}$ \\
\hline DIMI (Kunzel et al. 2011) & $\begin{array}{l}\text { Companheiro do aluno. Expressa as emoções felicidade, tristeza e companheirismo. A tristeza é expressa por DIMI } \\
\text { quando o aluno tem dificuldade, ao errar um exercício ou quando este não está gostando da interação. A felicidade é } \\
\text { expressa no momento que o aluno está gostando da interação e não apresenta dificuldades, acertando os exercícios. O } \\
\text { companheirismo é o estado padrão do agente DIMI durante a interação aluno/ambiente. }\end{array}$ \\
\hline MIKE (Lane et al. 2011) & $\begin{array}{l}\text { Ajuda os visitantes à Robot Park, uma exposição interativa para programação de computadores; usa animações e voz } \\
\text { sintetizada para interagir com os visitantes, dando-lhes várias formas de apoio ao longo da visita ao museu. }\end{array}$ \\
\hline $\begin{array}{l}\text { DIVALITE (Sansonnet et } \\
\text { al. 2012) }\end{array}$ & $\begin{array}{l}\text { Um framework para o desenvolvimento } W e b \text { baseado em ECA (Agentes Corporificados Conversacionais), do inglês } \\
\text { Embodied Conversational Agents. Dada uma página } W e b \mathrm{~W} \in \mathrm{W} \text {, o framework Divalite permite que o programador } \\
\text { integre um ou vários agentes nesta página para produzir uma página conversacional } \mathrm{W}^{*} \in \mathrm{W}^{*} \text {. Os agentes } \\
\text { implementados a partir do Divalite apresentam dimensões tais como personagens virtuais dotados de rica expressão } \\
\text { gráfica de emoções e o estudo do seu impacto nos usuários, capazes de reduzir a pesquisa do aluno gerada por carga } \\
\text { cognitiva das páginas } W e b . E \text { Expregado em aplicações como e-learning e jogos eletrônicos. }\end{array}$ \\
\hline $\begin{array}{l}\text { Coach Mike (Lane et al. } \\
\text { 2013) }\end{array}$ & $\begin{array}{l}\text { Coach Mike é um agente pedagógico animado que usa técnicas de tutores inteligentes para ajudar os alunos em } \\
\text { adquirir habilidades em programação básica de computadores. Entusiasma os alunos, dando-lhes feedback de suas } \\
\text { resoluções, falando-lhes sobre depuração, bem como explicando a função de específicos comandos de programação. }\end{array}$ \\
\hline
\end{tabular}




\begin{tabular}{c|l}
\hline $\begin{array}{c}\text { MInA (Rissoli; Santos, } \\
\text { 2013) }\end{array}$ & $\begin{array}{l}\text { MInA interage afetivamente com o usuário, priorizando a transmissão de aspectos afetivos e emocionais, através de } \\
\text { uma forte abordagem visual. Usa frases e metáforas que informa ao aluno a sua situação de aprendizagem, de forma } \\
\text { realista e motivadora, posicionando o agente como assistente e companheiro nos desafios educacionais. }\end{array}$ \\
\hline $\begin{array}{c}\text { KinSAR (Keren; Fridin, } \\
\text { 2014) }\end{array}$ & $\begin{array}{l}\text { O KinSAR (Kindergartem Social Assistiva Robot) é um robô que promove o desenvolvimento de crianças por meio da } \\
\text { interação social. O KindSAR ajuda a equipe educacional escolar no ensino de pensamento geométrico e na promoção } \\
\text { do desenvolvimento metacognitivo, envolvendo as crianças em atividades lúdicas interativas. }\end{array}$ \\
\hline $\begin{array}{c}\text { Oscar CITS (Latham et } \\
\text { al. 2014) }\end{array}$ & $\begin{array}{l}\text { Oscar CITS é um sofisticado ITS (Intelligent Tutoring Systems) que usa uma interface de linguagem natural para } \\
\text { permitir a alunos universitários construir seu próprio conhecimento através da discussão. Oscar CITS pretende imitar } \\
\text { um tutor humano, detectando e adaptando-se a estilos de aprendizagem de um indivíduo, enquanto dirige o tutorial de } \\
\text { conversação de forma dinâmica. }\end{array}$ \\
\hline
\end{tabular}

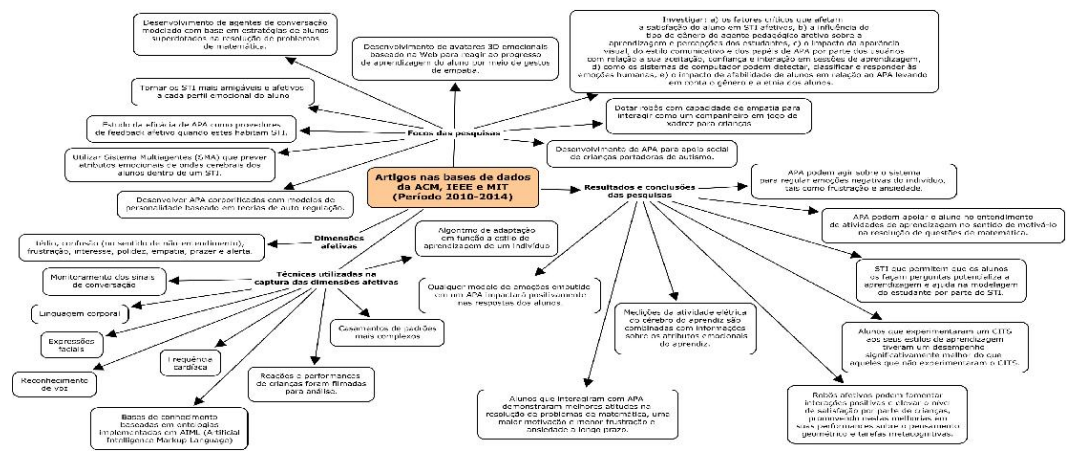

Figura 1: Aspectos identificados sobre APA nas bases da ACM, IEEE e MIT.

\section{Análises e discussões}

a) Constatou-se uma tendência, tanto no Brasil como no Mundo, investigar a afetividade na IHC e corporificação de comportamentos afetivos, podendo ser inferências e/ou simulação. b) o conceito de agentes pedagógicos tem se mantido como tema de estudo. Isso em virtude que os mesmos podem ser capazes de oferecer instruções personalizadas, aumentar a motivação dos estudantes, bem como são capazes de agirem por conta própria ou em conjunto com o professor. Entretanto, chama-se atenção que no estudo de APA, a nível de Brasil e do Mundo, carecem a distinção entre agente pedagógico, agente pedagógico afetivo e agente pedagógico animado, necessitando de uma distinção entre estes conceitos. c) Analisando-se os gráficos 1, 2 e 3, constatou-se que há um maior depósito de estudos na área de APA nas bases do mundo em relação às bases do Brasil. Salientando-se que a nível do Brasil, um maior depósito de APA encontram-se no SBIE, assim como a nível mundial há uma maior frequência nas bases do MIT. d) $\mathrm{O}$ estudo observou um padrão nas pesquisas de um foco para o desenvolvimento de APA para interagir com o indivíduo, ou seja, um processo de ensino e aprendizagem personalizado, e com isso o estudo não detectou foco que explorasse as percepções e ações destes agentes, bem como suas técnicas de desenvolvimento, que apoiassem sessões de aprendizagem colaborativa.

\section{Conclusões e direções futuras}

1) O emprego de APA vem se tornando útil no auxílio do processo de ensino e aprendizagem. 2) A vantagem do emprego de APA está na busca de melhorias dos processos de interação com os estudantes, identificando e inferindo suas ações, buscando se adaptar aos estilos cognitivos de cada um, de forma a ajudá-los em suas sessões de aprendizagem. 3) É possível que, se mais características de um professor humano forem corporificados aos APA, os resultados de aprendizagem podem ser mais 
eficazes. Já como direções futuras, pode-se destacar: a) Utilização de APA para apoiar a aprendizagem colaborativa. b) Explorar teorias da psicologia positiva, das características individuais e das instituições positivas centrado na prevenção e na promoção da saúde mental.

\section{Referências}

Bercht, M. Em direção a Agentes Pedagógicos com dimensões afetivas. Tese (Doutorado) - Porto Alegre: PPGC da UFFRGS, 2001.

Chatzara, K. et al. Emotional Interaction in e-Learning. Research on e-Learning and ICT in Education. Springer New York, 2012. 253-265.

Deters, J. I. et al. Bernardo - Agente Pedagógico do Sistema Tutor Inteligente Aplicado a Neurofisiologia. In: Anais do XVII Simpósio Brasileiro de Informática na Educação - SBIE - UNB/UCB, 2006.

De Oliveira, H. T. A, et al. "Dr. Pierre: Um Chatterbot com Intenção e Personalidade Baseado em Ontologias para Apoiar o Ensino de Psiquiatria." Anais do Simpósio Brasileiro de Informática na Educação. Vol. 1. No. 1. 2010.

Flick, Uwe. Introdução à metodologia de pesquisa: um guia para iniciantes. Porto Alegre: Penso, 2013.

Jaques, P. A. (2004). Using an Animated Pedagogical Agent to Interact Affectivelly with the Student. Programa de Pós-Graduação em Ciência da Computação. Porto Alegre, Universidade Federal do Rio Grande do Sul. PhD. Thesis, p. 228.

Johnson, W. L.; Rickel, J. STEVE: An animated pedagogical agent for procedural training in virtual environments. ACM SIGART Bulletin 8. 1-4 (1997): 16-21.

Johnson, W. L. et al. Animated pedagogical agents: Face-to-face interaction in interactive learning environments. Inernational Journal of Artificial Intelligence in Education 11.1 (2000): 47-78

Johnson, W. L. et al. Evolution of user interaction: the case of agent Adele. Proceedings of the 8th international conference on intelligent user interfaces, ACM, 2003.

Keren, G.; Fridin, M. "Kindergarten Social Assistive Robot (KindSAR) for children's geometric thinking and metacognitive development in preschool education: A pilot study." Computers in Human Behavior 35 (2014): 400-412.

Kunzel, M. F. et al. "DIMI 3D--Companion Agent in a Learning Virtual Environment." Agent Systems, their Environment and Applications (WESAAC), 2011 Workshop and School of. IEEE, 2011.

Lane, H. C., et al. "Intelligent tutoring goes to the museum in the big city: A pedagogical agent for informal science education." Artificial Intelligence in Education. Springer Berlin Heidelberg, 2011.

Lane, H. C. et al. The Effects of a Pedagogical Agent for Informal Science Education on Learner Behaviors and Self-Eficacy. In: Artificial Intelligence in Education. Springer Berlin Heidelberg, 2013.

Lepsen, E. et al. "Persona-Algo: Personalização dos Exercícios de Algoritmos auxiliados por um Agente Afetivo." Anais do Simpósio Brasileiro de Informática na Educação. Vol. 1. No. 1. 2010.

Latham, A. et al. "An adaptation algorithm for an intelligent natural language tutoring system."Computers \& Education 71 (2014): $97-110$.

Mello, G. et al. Implementando o Agente de Base de Domínio do Sistema Tutor Inteligente PAT2Math. In: XIII Ciclo de Palestras sobre Novas Tecnologias na Educação, 2009, Porto Alegre. RENOTE - Revista Novas Tecnologias na Educação, 2009.

OR, J. The development of emotional flexible spine humanoid robots. In: Advanced Robotics Systems, 2008).

Picard, R. W. Affective Computing. Cambridge, Massachusetts: MIT Press, 1997, 292p.

Rissoli, V. R. V.; Santos, G. A. "O Agente Pedagógico Animado MInA." Anais do Simpósio Brasileiro de Informática na Educação. Vol. 24. No. 1. 2013

Sansonnet, J. P. et al. Developing Web fully-integrated conversational assistant agents. Proceedings of the 2012 ACM Research in Applied Computation Symposium. ACM, 2012.

Santos, C. T. dos, et al. "DÓRIS - Um Agente de Acompanhamento Pedagógico em Sistemas Tutores Inteligentes."Anais do Simpósio Brasileiro de Informática na Educação. Vol. 1. No. 1. 2001.

Seffrin, H., et al. "Resolvendo equações algébricas no STI PAT2Math." Anais do Simpósio Brasileiro de Informática na Educação. Vol. 1. No. 1. 2010 .

Silva, C. G.; Figueiredo, V. F. Ambiente Virtual de Aprendizagem: comunicação, interação e afetividade na EAD. Revista Aprendizagem em EAD. V01, 2012.

Strauss, M.; Kipp, M. "ERIC: a generic rule-based framework for an affective embodied commentary agent. Proceedings of the 7th International Joint Conference on Autonomous Agents and Multiagent Systems. v. 1. International Foundation for Autonomous Agents and Multiagent Systems, 2008 